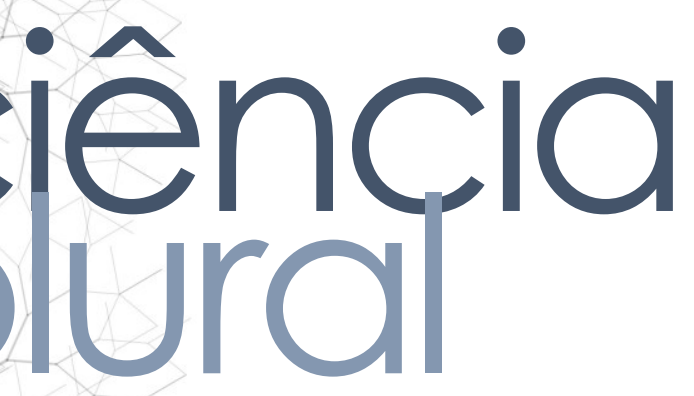

\title{
AVALIAÇÃO DO NÍVEL DE CONHECIMENTO DOS PACIENTES ATENDIDOS NA ATENÇÃO BÁSICA SOBRE A PREVENÇÃO DOS CÂNCERES DE CABEÇA E PESCOÇO
}

Assessment of the level of knowledge of patients treated in primary care on the prevention of head and neck cancers

Evaluación del nivel de conocimiento de los pacientes atendidos en cuidados básicos sobre la prevención del cáncer de cabeza y cuello

Arlon Néry do Nascimento • Cristo Faculdade do Piauí - CHRISFAPI • E-mail: arlonnerys3@gmail.com

Taynara Lorrana Oliveira Araújo • Cristo Faculdade do Piauí - CHRISFAPI • E-mail: tay.nara13@outlook.com

Lyandra Ellen Bezerra Meneses • Cristo Faculdade do Piauí - CHRISFAPI • E-mail: ellenlyandra15@gmail.com

Mayane Carneiro Alves Pereira • Universidade Federal do Piauí - UFPI • E-mail: mayanealves@hotmail.com

Autor correspondente:

Arlon Néry do Nascimento• E-mail: arlonnerys3@gmail.com 


\section{RESUMO}

Introdução: O câncer de cabeça e pescoço é um conjunto de neoplasias que atingem o trato aero digestivo superior. Essas neoplasias apresentam sintomatologia variada, que depende da localização do tumor, gravidade e tempo de ocorrência. Objetivo: Avaliar o nível de conhecimento da população, usuária da atenção básica, acerca da prevenção e tratamento dos Cânceres de Cabeça e Pescoço. Metodologia: Trata-se de um estudo de campo transversal descritivo, em que se coletou informações acerca do conhecimento sobre cânceres de cabeça e pescoço, informações sociodemográficas, histórico familiar, características clínicas e de tratamentos. A coleta foi realizada em julho de 2019, em três Estratégias de Saúde da Família da cidade de Piripiri, Piauí. A pesquisa foi submetida e aprovada pelo Comitê de Ética em Pesquisa com o Parecer $\mathrm{n}^{\mathrm{o}}$ 3.221.522. Resultados e discussão: A maioria dos entrevistados foram mulheres solteiras com idade média de 44,4 anos e de baixa renda. Dentre os questionamentos realizados, grande parte não soube responder questões sobre conhecimentos gerais a respeito dessas enfermidades. Outro fator importante é a baixa procura por serviços de saúde. Conclusões: Observou-se que os entrevistados apresentam desinformação a respeito da prevenção e tratamento desse grupo de cânceres, sendo necessário o fortalecimento de ações de educação popular em saúde.

Palavras-Chave: Atenção Primária à Saúde. Neoplasias bucais. Neoplasias orofaríngeas. Profilaxia.

\section{ABSTRACT}

Introduction: Head and neck cancer is a group of neoplasms that affect the upper aerodigestive tract. These neoplasms have varied symptoms, depending on the location of the tumor, severity and time of occurrence. Objective: To assess the level of knowledge of the population, users of primary care, about the prevention and treatment of Head and Neck Cancer. Methodology: This is a descriptive crosssectional study, in which information about knowledge about head and neck cancers, sociodemographic information, family history, clinical and treatment characteristics was collected. The collection was carried out in July 2019, in three Family Health Strategies in the city of Piripiri, Piauí. The research was submitted and approved by the Research Ethics Committee with Opinion No. 3,221,522. Results and discussion: Most of the interviewees were single women with an average age of 44.4 years and low income. Among the questions asked, most of them did not know how to answer questions about general knowledge about these diseases. Another important factor is the low demand for health services. Conclusions: It was observed that the interviewees have misinformation regarding the prevention and treatment of this group of cancers, and it is necessary to strengthen popular health education actions.

eywords: Primary Health Care. Oral neoplasms. Oropharyngeal neoplasms. rophylaxis. 


\section{RESUMEN}

Introducción: El cáncer de cabeza y cuello es un grupo de neoplasias que afectan el tracto aero digestivo superior. Estas neoplasias tienen síntomas variados, que dependen de la ubicación del tumor, la gravedad y el momento de aparición. Objetivo: Evaluar el nivel de conocimiento de la población, usuarios de la atención primaria de salud, sobre la prevención y el tratamiento del Cáncer de Cabeza y Cuello. Metodología: Se trata de un estudio descriptivo transversal, en el que se recopiló información sobre conocimientos sobre cánceres de cabeza y cuello, información sociodemográfica, antecedentes familiares, características clínicas y de tratamiento. La recolección se realizó en julio de 2019, en tres Estrategias de Salud de la Familia en la ciudad de Piripiri, Piauí. La investigación fue sometida y aprobada por el Comité de Ética en Investigación con Opinión No. 3.221.522. Resultados y discusión: La mayoría de los encuestados eran mujeres solteras con una edad promedio de 44,4 años y bajos ingresos. Entre las preguntas formuladas, la mayoría de ellos no sabía cómo responder preguntas sobre conocimientos generales sobre estas enfermedades. Otro factor importante es la baja demanda de servicios de salud. Conclusiones: Se observó que los entrevistados tienen desinformación sobre la prevención y tratamiento de este grupo de cánceres, y es necesario fortalecer las acciones de educación popular en salud.

Palabras clave: Atención Primaria de Salud. Neoplasias bucales. Neoplasias orofaríngeas. Profilaxis. 


\section{Introdução}

A palavra câncer de cabeça e pescoço (CCP) refere-se a um conjunto de neoplasias heterogêneas que atingem o trato aero digestivo superior, região essa responsável por funções básicas como a respiração, deglutição e comunicação. Os tumores podem atingir regiões como a cavidade oral, lábios, laringe, faringe, cavidade nasal e tireoide ${ }^{1}$.

O câncer é considerado um grande problema atual para a saúde pública, sendo a segunda principal causa de morte no Brasil, estimativas mostram a ocorrência de 11.200 casos novos de câncer da cavidade oral no sexo masculino e 3.500 no sexo feminino, apenas no biênio 2018-2019. Esses valores correspondem a um risco estimado de 10,86 casos novos a cada 100 mil homens, ocupando a quinta posição entre os cânceres; e de 3,28 para cada 100 mil mulheres, sendo o $12^{\circ}$ mais frequente entre todos os cânceres para esse gênero ${ }^{2}$.

Vários fatores podem estar interligados ao desenvolvimento de $\mathrm{CCP}$, dentre eles a questão socioeconômica, etilismo, tabagismo, exposição excessiva ao sol, infecções virais, questões ocupacionais e a ocorrência de um tumor em algum outro local da cabeça ou pescoço são os predominantes em sua etiologia. Esse tipo de câncer apresenta uma sintomatologia variada, depende da localização do tumor, gravidade e duração. Seu rastreamento ocorre, majoritariamente, de maneira ocasional e não atrelado a aparição de sinais prévios ${ }^{3,4}$.

Considerado de diagnóstico simples para a equipe médica, o CCP apresenta-se bem característico no exame clínico, sendo de fácil palpação e visualização. Entretanto, apesar desse fator, na maior parte dos enfermos seu diagnóstico é tardio, condição explicada pela falta de sintomatologia expressiva e pelo acompanhamento irregular ao profissional de saúde. Destaca-se que o período de tempo entre a consulta inicial e o tratamento é longo, por esse motivo seu diagnóstico geralmente ocorre em estádios já vançados, e quanto maior a demora para a confirmação da doença, pior é seu stadiamento e mais complexo o estabelecimento do tratamento, sendo necessário ma terapêutica mais agressiva 5 .

A falta de conhecimento populacional sobres os CCP são razões teorizadas pelas is esse grupo de doenças é diagnosticado em um estágio de alto risco, sendo um 
fator contribuinte para progressão de um prognóstico não desejável. Outra razão que pode afetar a saúde de um indivíduo é seu nível de alfabetização, sendo um importante artifício utilizado para compreensão de quando deve ser tomada decisões na procura de um serviço de saúde, com o intuito de prevenir ou tratar qualquer demanda específica 6 .

Atualmente a incidência de CPP tem aumentado, tratando-se de tumores que estão ligados com altos índices de recidiva e também a uma elevada mortalidade. $\mathrm{O}$ tratamento primário depende de topografia, e a cirurgia é fundamental em muitas situações para cura dos pacientes. Em muitos casos o tratamento gera sequelas funcionais ou estéticas no corpo dos pacientes, que podem ser definitivas na vida dos mesmos. A família também apresenta um déficit de conhecimento em relação aos cuidados de seus entes, por esse motivo, também necessitam de uma atenção, para que recuperação do paciente seja mais eficaz ${ }^{7}$.

Diante aos dados expostos e reconhecendo a relevância desta temática, o presente estudo tem o objetivo de avaliar o nível de conhecimento da população, usuária da atenção básica, acerca da prevenção e tratamento dos Cânceres de Cabeça e Pescoço.

\section{Metodologia}

\section{Desenho do estudo e amostra}

Trata-se um estudo de campo transversal descritivo, a partir de um questionário que abordou as principais características sobre os Cânceres de Cabeça e Pescoço, em pacientes atendidos em serviço de Atenção Básica de Saúde no município de PiripiriPI.

Os critérios de inclusão foram indivíduos de ambos os sexos, com idade superior a 18 anos, usuários da Estratégias de Saúde da Família (ESF) e portadores de Cartão de Saúde do Sistema Único de Saúde (SUS). Como critérios de exclusão os pacientes que não apresentaram um bom desempenho cognitivo através do Mini Exame do Estado Mental e que não conseguisse fornecer todas as informações solicitadas.

leta de Dados

Elaborou-se um questionário no intuito de colher informações sociodemográficas o, idade, estado civil, profissão, nível escolar, número de habitantes na residência 
e renda salarial), pessoais sobre histórico familiar de câncer, além das características clínicas e de tratamento relacionados aos CCP. Para finalizar, buscou-se informações sobre educação popular em saúde e nível de comunicação dos funcionários da Atenção Básica com os pacientes sobre prevenção e tratamento dos CCP.

O procedimento para a coleta dos dados foi realizado em duas ESF na cidade de Piripiri -PI (ESF 18 - Bairro Centro e EFS 17 - Bairro Caixa d'água) em um período no mês de julho 2019. Durante toda a pesquisa foi mantido o sigilo e o anonimato dos pesquisados, a coleta ocorreu de maneira individualizada, em uma sala devidamente reservada na ESF. Os pesquisadores foram devidamente treinados no intuito de obter uma uniformidade das informações.

\section{Considerações éticas}

Antes de iniciada a coleta a pesquisa foi submetida e aprovada pelo Comitê de Ética em Pesquisa da Universidade Federal do Piauí de acordo com o Parecer $\mathrm{n}^{\mathrm{o}}$ 3.221.522. Todos os pesquisados concordaram em participar do estudo e assinaram o Termo de Consentimento Livre e Esclarecido antes de iniciada a coleta de dados.

\section{Análise estatística}

O conteúdo do estudo foi coletado a partir de formulários que foram transferidos para um banco de dados preparado no aplicativo Microsoft Office Excel@. Em seguida, para análise descritiva utilizou-se valores absolutos, médias, desvio padrão e porcentagem; de acordo com a necessidade da variável. Para um melhor entendimento os resultados obtidos foram expressos em quadros e tabelas.

\section{Resultados}

O questionário foi aplicado em 30 pacientes maiores de 18 anos que apresentassem um bom cognitivo, foram excluídos três usuários pois não souberam fornecer todas as informações solicitadas, permaneceram no estudo 27 pesquisados. Para um maior entendimento, os dados foram expostos em diferentes tabelas.

Inicialmente analisou-se os dados sociodemográficos dos participantes. Nessa ndição observou-se que a maioria são do sexo feminino $(85,2 \%)$, com média de 43,21 os e com formação em ensino médio completo 7 ou incompleto 2. Os demais dados ofissão, renda, número de habitantes) estão expostos na Tabela 1. 
Tabela 1: Aspectos sociodemográficos dos usuários atendidos na atenção primária a saúde. Piripiri-PI, 2019.

\section{VARIÁVEIS}

IDADE MÉDIA

\section{SEXO}

Feminino

Masculino

ESTADO CIVIL

Solteiro (a)

Casado (a)

Divorciado (a)

Viúvo (a)

ESCOLARIDADE

Analfabeto

EF (in)completo

EM (in)completo

ES (in)completo

PROFISSÃO

Dono (a) de Casa

Estudante

Professor (a)

Vendedor (a)

Outros

Aposentado (a)

HABITANTES/ DOMICÍLIO

RENDA FAMILIAR

$<1$ salário mínimo

a 3 salários mínimos

a 6 salários mínimos

6 salários mínimos

\section{VALORES OBTIDOS}

$44,4( \pm 17,7)$ anos

$23(85,2 \%)$

$4(14,8 \%)$

$12(44,5 \%)$

$11(40,7 \%)$

$3(11,1 \%)$

$1(3,7 \%)$

$0(0 \%)$

$8(29,7 \%)$

$10(37 \%)$

$9(33,3 \%)$

$14(51,9 \%)$

$2(7,4 \%)$

$2(7,4 \%)$

$2(7,4 \%)$

$3(11,1 \%)$

$4(14,8 \%)$

$4,2( \pm 1,4)$

7 (25,9\%)

$19(70,4 \%)$

$1(3,7 \%)$

$0(0 \%)$

enda: EF: ensino fundamental; EM: ensino médio, ES: ensino superior.

te: Próprio Autor, 2020. 
Quando avaliado o histórico de câncer observou-se que os 27 entrevistados desconhecem como é realizado o diagnóstico dos cânceres de cabeça e pescoço, assim totalizando (100\%). Entretanto, ao abordar sobre a existência da patologia em suas famílias, 18 (66,7\%) pessoas afirmam que não há histórico familiar da doença e apenas $9(33,3 \%)$ relataram que houve sim casos de parentes que foram diagnosticados com câncer.

Na Tabela 2 estão expostos os questionamentos realizados nas ESF a respeito de determinados dados relacionados aos cânceres. Diante disso, foi observado que a maioria desconhece as principais informações ligadas a promoção e prevenção do câncer de cabeça e pescoço sendo os seguintes: sintomas (24-88,9\%); fatores de risco (17-63\%); prevenção (15-55,6\%); diagnóstico (21-77,8\%) e impactos da cirurgia no organismo humano (18-66,7 \%). Todavia, quando foram questionados sobre os impactos do tratamento conservador como a quimioterapia/ radioterapia foi possível notar que a grande parte dos usuários souberam responder esse quesito 18 (66,7\%).

Tabela 2: Verificação do nível de conhecimento dos pacientes atendidos na ESF 18Bairro Centro e ESF 17- Bairro Caixa d'água. Piripiri-PI, 2019.

\begin{tabular}{lcc}
\hline \multicolumn{1}{c}{ QUESTIONAMENTO } & SIM & NÃO \\
\hline Você conhece os sintomas dos CCP? & $3(11,1 \%)$ & $24(88,9 \%)$ \\
Você conhece os fatores de risco dos CCP? & $10(37 \%)$ & $17(63 \%)$ \\
Você sabe como prevenir os CCP? & $12(44,4 \%)$ & $15(55,6 \%)$ \\
Você sabe como é feito o diagnóstico dos CCP? & $6(22,2 \%)$ & $21(77,8 \%)$ \\
Você conhece os impactos da cirurgia curativa para & $9(33,3 \%)$ & $18(66,7 \%)$ \\
CCP? & & \\
Você $\quad$ conhece $\quad$ impactos da & $18(66,7 \%)$ & $9(33,3 \%)$ \\
quimioterapia/radioterapia no tratamento dos CCP? & \\
\hline
\end{tabular}

Legenda: ESF: estratégia de saúde da família, CCP: câncer de cabeça e pescoço. Fonte: Próprio Autor, 2020.

Em relação a interação dos pesquisados com a equipe de saúde é possível afirmar a população faz uso dos serviços ofertados pela ESF com uma baixa frequência, curando atendimentos apenas uma vez ao mês, em média (15-55,6\%). Ao perguntar 
se os profissionais falavam sobre os cânceres e seus respectivos fatores de risco, a maioria demonstraram que não $(21-77,8 \%)$, como também não discutiam sobre os meios de prevenção com os pacientes (17-63\%). Além do mais, o tipo de câncer mais falado foi o de mama (10) seguido pelo o de próstata (3).

\section{Discussão}

\section{A realidade da população adscrita}

Os dados levantados nesta pesquisa, demonstram que a população apresenta baixa renda, sendo que $(70,4 \%)$ dos entrevistados recebem entre um a três salários mínimo, e tem por maioria o sexo feminino $(85,2 \%)$, esta condição pode estar atrelada ao fato de as mulheres procurarem, com maior frequência, o serviço de atenção primária à saúde ${ }^{8}$. Essa situação é reforçada pelo fato de indivíduos do sexo masculino estarem mais ligado a hábitos curativos e menos aos de prevenção, quando comparado

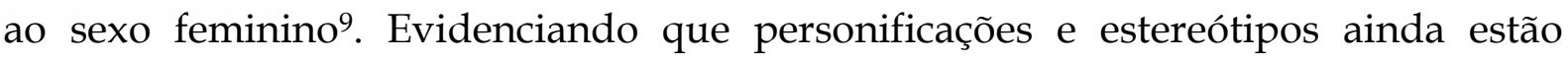
fortemente interligados ao gênero e à população em geral, tais como: “Força está atrelada aos homens"; "mulheres necessitam de maior cuidado", "o SUS está atrelado a status de pobreza" 10 .

A Política Nacional de Atenção Integral a Saúde do Homem (PNAISH), tem como objetivo principal melhorar as condições de saúde para população masculina, focando em reduzir a morbidade e mortalidade, analisando os fatores de risco para esse grupo e oferecendo condições para aumentar o serviço de acesso em saúde ${ }^{11}$. Entretanto, pesquisas recentes demonstram que homens tendem a ter hábitos de vidas menos saudáveis e maior presença de doenças crônicas. Dessa forma, pode-se dizer que adentram no sistema de saúde por meio da atenção especializada, pois não tem o hábito de procurar a atenção primária como as mulheres, agravando assim a morbidade desta população ${ }^{12}$.

No Brasil a saúde tem o status de direito social, para assegurá-la, foi criado o SUS, e visa atendimento universal e integral da população. Entretanto, a realidade rece uma série de obstáculos prático, além do estigma que o associa o SUS apenas ppulação de baixa renda. Uma significativa parcela da população não aciona o SUS 
em condições de promoção, deixando para utilizá-lo apenas em situações de adoecimento. Atingir essa parte da população é um desafio para a Saúde Pública13.

O estado civil dos entrevistados mostrou-se diversificado e sem diferenças discrepantes. No entanto, observou-se uma leve preponderância ao uso por pessoas solteiras $(44,5 \%)$ e do sexo feminino $(85,2 \%)$, com idade média de $44,4( \pm 17,7)$ anos. Destacando a desconstrução da cultura do casamento, com construção do lar, do reflexo social que o ser casado agrega, ou seja, de manutenção das relações sociais de prestação de serviços e cuidados aos membros da família ${ }^{14}$.

Os participantes relataram um grupo familiar com média de 4,2 $( \pm 1,4)$ habitantes por casa, isso mostra que a população analisada ainda adota um grupo familiar maior. De acordo com dados do último senso do IBGE (2010), o número de jovens residindo com os pais na faixa dos 25 aos 30 anos, cresceu de 3,3 para 4,7 milhões. O prolongamento da convivência familiar é uma tendência em perspectiva crescente hoje em dia, acredita-se então que a maioria dos entrevistados por se apresentarem mais velhos, ainda residem com os filhos em sua própria casa. Por conta da comodidade dos filhos, pois os pais já podem apresentar uma renda mais estável, como também a divisão de despesas do entre um grupo familiar maior se torna mais cômodo ${ }^{15}$. Vivência, experimentação e conhecimento acerca dos Cânceres de Cabeça e Pescoço

O recebimento de um diagnóstico de câncer provoca inúmeros sentimentos, preocupações e inquietações em seus acometidos. Em seu contexto, o amanhã tornase obscuro, muitas vezes sem perspectivas, pois a ameaça da morte parece tornar-se mais próxima quando o diagnóstico se encontra estabelecido ${ }^{16}$. Um diagnóstico indesejado pode sempre afetar o funcionamento de um grupo familiar. Os valores, a história da família, o conhecimento prévio e as suas expectativas em relação ao tratamento podem intervir na forma de lidar com a doença e nos cuidados para com o membro enfermo ${ }^{17}$.

Dentre os entrevistados cerca de 77,8\%, não sabe como é realizado o diagnóstico, cerca $66,7 \%$ não conhece os impactos da cirurgia curativa de cânceres de CCP. Isso stra que em função de seu estigma social e de sua associação com a morte, dor e tamentos invasivos, o câncer é uma enfermidade que provoca temor social, já que co se conhece sobre o diagnóstico e pouca se houve falar de curas. Por esse motivo, 
pesquisas investigam o impacto do diagnóstico de câncer não apenas no paciente, mas também em sua família. Esses sentimentos surgem mesmo com o alcance de cura e da sobrevida de muitos tipos de câncer, através dos avanços técnico-científicos alcançados nas últimas décadas ${ }^{18}$.

A própria palavra câncer está relacionada com implicações negativas da doença, a sua pronúncia é reprimida para algumas pessoas, em uma tentativa de afastá-lo ou nega-lo do sofrimento, angústias e medo que a enfermidade carrega ${ }^{19}$. Durante a coleta de dados observou-se que a maioria não conhece os sintomas $(88,9 \%)$, fatores de risco (63\%) e meios de prevenção (55,6\%). A demanda de informações sobre a doença, apresenta-se crescente tanto para os pacientes como para os familiares. Entender os casos e de suma importância para a tomada de decisões assertivas sobre o tratamento, é essencial que eles tenham conhecimento suficiente para fazê-lo. Entretanto, adquirir essas informações é um processo complexo e extenso que depende de vários fatores como cultura, mitos, crenças e religiões ${ }^{20}$.

Destaca-se também que cerca de 66,7\% dos entrevistados afirmaram conhecer os impactos da quimioterapia. Sendo reconhecida principalmente pelos seus efeitos colaterais, como vômitos, alopecia, náuseas, constipação ou diarreia. Associadas a isso também ocorrem alterações na aparência física, dificuldade para manter um vínculo empregatício e as relações interpessoais que comprometem a qualidade de vida das pessoas no âmbito físico, psíquico e social. Enaltecendo principalmente o que se ver nas mídias ${ }^{19}$.

\section{Educação popular e câncer na Estratégia de Saúde da Família}

Pode-se afirmar que a frequência de pessoas que procuram os serviços de saúde é baixa (15-55,6\%), sendo que essa procura acontece de forma mensal. A maioria deles optam por procurar assistência apenas quando sentem alguma desordem em seu organismo, no entanto, esse pensamento acaba contribuindo para a que a doença se instale e evolua progressivamente. Esse comportamento ligado a prevenção está relacionado a fatores psicológicos, sociais, ambientais e culturais. A falta de nformação por parte da população pode influenciar positiva ou negativamente no gnóstico e prognóstico da patologia 21. 
Essa desinformação pode estar atrelada ao nível socioeconômico dos indivíduos, além do mais, a falta de discussão dos profissionais de saúde com os seus pacientes, por meio de um processo de educação permanente, complica esse quadro. Vale lembrar que todos os profissionais que atuam na ESF são fundamentais para o rastreamento desse tipo de câncer, contudo aquele que está intimamente relacionada com essa demanda é o profissional dentista, pois, a partir da rotina dos atendimentos os mesmos tem o dever de identificar o câncer bucal ou fatores relacionados ao seu surgimento. Um dos campos de atenção primária é o diagnóstico precoce, facilitado por meio do autoexame bucal, que deve ser repassado para o paciente realizar constantemente em seu domicílio e não só quando for a consulta odontológica. Todavia, isso não está acontecendo, pois os cirurgiões-dentistas não costumam ensinálos, pelo o fato da ausência de capacitação ou por não acharem necessário tal procedimento $^{21,22}$

Diante disso, os cânceres mais debatidos entre os pacientes foram o de mama (10) e o de próstata (3). As campanhas relacionadas a educação em saúde em neoplasias estão voltadas para esses casos. Com isso, é importante reforçar a educação popular em saúde e a educação permanente, entre os profissionais. A educação permanente é um novo conceito na área da promoção em saúde, dessa forma, mesmo fora das graduações, será possível incluir debates acerca da Saúde Coletiva e de questões de saúde relacionadas ao SUS, além de possibilitar a qualificação desses profissionais²3.

\section{Conclusões}

Com esta pesquisa, conclui-se que o sexo feminino busca com mais frequência aos serviços de atenção primária, no entanto ainda há uma grande demanda de informações desconhecidas relacionadas ao câncer de cabeça e pescoço para os usuários da ESF. Diante disso, é importante implementar ações de orientações sobre a atologia em questão, por parte dos profissionais da atenção básica, já que se trata de tipo de câncer pouco abordado. Desse modo, haverá uma minimização dessa sência de conhecimentos na sociedade, melhorando assim seu conhecimento dentro rea de promoção em saúde, o que possibilita fornecer melhores cuidados de saúde 
e melhor chance de um diagnóstico precoce. Vale ressaltar a necessidade de mais pesquisas relacionadas a essa enfermidade.

\section{Referências}

1- Avelar JMP, Nicolussi AD, Toneti BF, Sonobe HM, Sawada NO. Fadiga em pacientes com câncer de cabeça e pescoço em tratamento radioterápico: estudo prospectivo. Rev. Latino-Am. Enfermagem. 2019; [acesso em 2020 jan 05]; 27:e3168. Disponível em: https:// doi.org/10.1590/1518-8345.2813-3168.

2- Instituto Nacional de Câncer José Alencar Gomes da Silva. Estimativa | 2018 [Internet]. Rio de janeiro: INCA; 2018. Incidência de Câncer no Brasil. [acesso em 2020 jan 05]. Disponível em: https://www.inca.gov.br/estimativa/2018.

3- Ferraz CAG, Rezende G, De Carlo MMRP. Uso de tecnologia de comunicação alternativa na avaliação da qualidade de vida de pacientes com câncer de cabeça e pescoço. Cad. Bras. Ter. Ocup. [acesso em 2020 jan 05]; 2019;27(1):61-71. Disponível em: http://dx.doi.org/10.4322/2526-8910.ctoao1761.

4- Ribeiro ILA, Medeiros JJ, Rodrigues LV, Valença AMG, Lima Neto EA. Fatores associados ao câncer de lábio e cavidade oral. Rev. bras. epidemiol. [acesso em 2020 jan 05]; 2015;18(3):618-629. Disponível em: https:/ / doi.org/10.1590/19805497201500030008 .

5- Felippu AWD, Freire EC, Silva RA, Guimarães AV, Dedivitis RA. Impact of delay in the diagnosis and treatment of head and neck cancer. Braz J. Otorrinolaringol. [acesso em 2020 jan 05]; 2016;82(2):140-143. Disponível em: https://doi.org/10.1016/j.bjorl.2015.10.009.

6- Jesse MT, Fei N, Goldstein E, Rakitin I, Shama L, Hall F, et al. Head and neck cancer screenings and human papillomavirus knowledge across diverse suburban and urban populations. American Journal of Otolaryngology. [acesso em 2020 jan 05]; 2015;36(2):223-229. Disponível em: https://doi.org/10.1016/j.amjoto.2014.10.037.

7- Rigoni L, Bruhn RF, Cicco R, Kanda JL, Matos LL. Déficit de qualidade de vida em pacientes com câncer de cabeça e pescoço e seus cuidadores: um estudo comparativo. Braz J. Otorrinolaringol. [acesso em 2020 jan 05]; 2016;82(6):680-686. Disponível em: https://doi.org/10.1016/j.bjorl.2015.12.012.

Botton A, Cúnico SD, Strey MN. Diferenças de gênero no acesso aos serviços de ưde: problematizações necessárias. Mudanças-Psicologia da Saúde. [acesso em 20 jan 05]; 2017;25(1):67-72. Disponível em: http://dx.doi.org/10.15603/21769/mud.v25n1p67-72. 
9- Machin R, Couto MT, Silva GSND, Schraiber LB, Gomes R, Figueiredo WS, et al. Concepções de gênero, masculinidade e cuidados em saúde: estudo com profissionais de saúde da atenção primária. Ciência \& Saúde Coletiva. [acesso em 2020 jan 20]; 2011;16(11):4503-4512. Disponível em:

https://www.scielosp.org/pdf/csc/2011.v16n11/4503-4512/pt.

10- Bertolini DNP, Simonetti JP. O gênero masculino e os cuidados de saúde: a experiência de homens de um centro de saúde. Esc Anna Nery. [acesso em 2020 jan 20]; 2014;18(4):722-727. Disponível em: DOI:10.5935/1414-8145.20140103.

11- Schwarz E, Gomes R, Couto MT, Moura EC, Carvalho SA, Silva SFC. Política de saúde do homem. Rev. Saúde Pública. [acesso em 2020 jan 20]; 2012; 46:108-116. Disponível em: https:/ / doi.org/10.1590/S0034-89102012005000061.

12- Bidinotto DNPB, Simonetti JP, Bocchi SCM. A saúde do homem: doenças crônicas não transmissíveis e vulnerabilidade social. Rev. Latino-Am. Enfermagem. [acesso em 2020 jan 20]; 2016;24:e2756. Disponível em: https:/ / doi.org/10.1590/15188345.0735.2756.

13- Osorio RG, Servo LMS, Piola SF. Necessidade de saúde insatisfeita no Brasil: uma investigação sobre a não procura de atendimento. Ciência \& Saúde Coletiva. [acesso em 2020 jan 20]; 2011;16(9):3741-3754. Disponível em:

https://www.scielosp.org/pdf/csc/2011.v16n9/3741-3754/pt.

14- Levorato CD, Mello LMD, Silva ASD, Nunes AA. Fatores associados à procura por serviços de saúde numa perspectiva relacional de gênero. Ciência \& Saúde Coletiva. [acesso em 2020 jan 20]; 2014;19(4):1263-1274. Disponível em: DOI: 10.1590/1413-81232014194.01242013.

15- Gallagher IM, Carneiro, TF, Henriques, CR. Planos para o futuro: percepções de filhos adultos coabitantes com os pais. Revista da SPAGESP. [acesso em 2020 jan 20]; 2013;14(2):4-18. Disponível em:

http:/ / pepsic.bvsalud.org/scielo.php?script=sci_arttext\&pid=S167729702013000200002.

16- Salci, MA, Marcon SS. Enfrentamento do câncer em família. Texto \& ContextoEnfermagem. [acesso em 2020 jan 20]; 2011;20(SPE):178-186. Disponível em: https:/ / doi.org/10.1590/S0104-07072011000500023.

17- Farinhas GV, Wendling MI, Dellazzana-Zanon LL. Impacto psicológico do diagnóstico de câncer na família: um estudo de caso a partir da percepção do idador. Pensando famílias. [acesso em 2020 jan 20]; 2013;17(2):111-129. Disponível : http:/ / pepsic.bvsalud.org/pdf/penf/v17n2/v17n2a09.pdf.

Scannavino CSS, Sorato DB, Lima MP, Franco AHJ, Martins MP, Morais Júnior et al. Psycho-oncology: the psychologist in the Barretos Cancer Hospital. 
Psicologia USP. [acesso em 2020 jan 20]; 2013;24(1):35-53. Disponível em: https://doi.org/10.1590/S0103-65642013000100003.

19- Wakiuchi J, Oliveira DC, Marcon, SS, Oliveira MLF, Sales CA. Sentidos e dimensões do câncer por pessoas adoecidas - análise estrutural das representações sociais. Revista da Escola de Enfermagem da USP. [acesso em 2020 fev 27]; 2020;54. Disponível em: http:/ / dx.doi.org/10.1590/S1980-220X2018023203504.

20- Lima M, Irigaray T. Lócus de controle, escolaridade e conhecimento sobre a doença em pacientes oncológicos. Psicologia, Saúde \& Doenças. [acesso em 2020 jan 20]; 2019;20(3):659-669. Disponível em: http:/ / dx.doi.org/10.15309/19psd200309.

21- Souza LRB, Ferraz KD, Pereira NS, Martins, MV. Conhecimento acerca do câncer bucal e atitudes frente à sua etiologia e prevenção em um grupo de horticultores de Teresina (PI). Revista Brasileira de Cancerologia. [acesso em 2020 jan 20]; 2012;58(1):31-39. Disponível em:

https://rbc.inca.gov.br/revista/index.php/revista/article/view/633.

22- Martins AMEBL, Barreto SM, Santos-Neto PE, Sá MAB, Souza JGS, Haikal DS, et al. Maior acesso à informação sobre como prevenir o câncer bucal entre idosos assistidos na atenção primária à saúde. Revista Ciência \& Saúde Coletiva. [acesso em 2020 jan 20]; 2015;20(7):2239-2253. Disponível em: https:/ / doi.org/10.1590/141381232015207.15272014 .

23- Amaral MCS, Pontes AGV, Silva JV. O ensino de Educação Popular em Saúde para o SUS: experiência de articulação entre graduandos de enfermagem e Agentes Comunitários de Saúde. Interface-Comunicação, Saúde, Educação. [acesso em 2020 jan 20]; 2014; 18:1547-1558, 2014. Disponível em: https:/ / doi.org/10.1590/180757622013.0441 . 